\title{
2 Differential effects of redox conditions on the decomposition of litter and soil organic matter
}

3 Yang Lin ${ }^{1,2^{*}}$, Ashley N. Campbell ${ }^{3}$, Amrita Bhattacharyya ${ }^{3,4}$, Nicole DiDonato ${ }^{5}$, Allison M. Thompson ${ }^{5}$, Malak M.

$4 \quad$ Tfaily $^{5,6}$, Peter S. Nico ${ }^{4}$, Whendee L. Silver ${ }^{1^{*}}$, and Jennifer Pett-Ridge ${ }^{3^{*}}$

$5{ }^{1}$ Department of Environmental Science, Policy, and Management, University of California, Berkeley, California

$6 \quad 94720$

$7 \quad{ }^{2}$ Department of Soil and Water Sciences, University of Florida, Gainesville, Florida 32611

$8 \quad{ }^{3}$ Physical and Life Sciences Directorate, Lawrence Livermore National Laboratory, Livermore, California 94550

$9 \quad{ }^{4}$ Earth Sciences Division, Lawrence Berkeley National Laboratory, Berkeley, California 94720

$10{ }^{5}$ Environmental Molecular Sciences Laboratory, Pacific Northwest National Laboratory, Richland, Washington

1199352

$12{ }^{6}$ Department of Environmental Science, University of Arizona, Tucson, Arizona 85719

13 Correspondence to: Yang Lin (ylin2@ufl.edu), Whendee L. Silver (wsilver@berkeley.edu), and Jennifer Pett-Ridge 14 (pettridge2@1lnl.gov) 

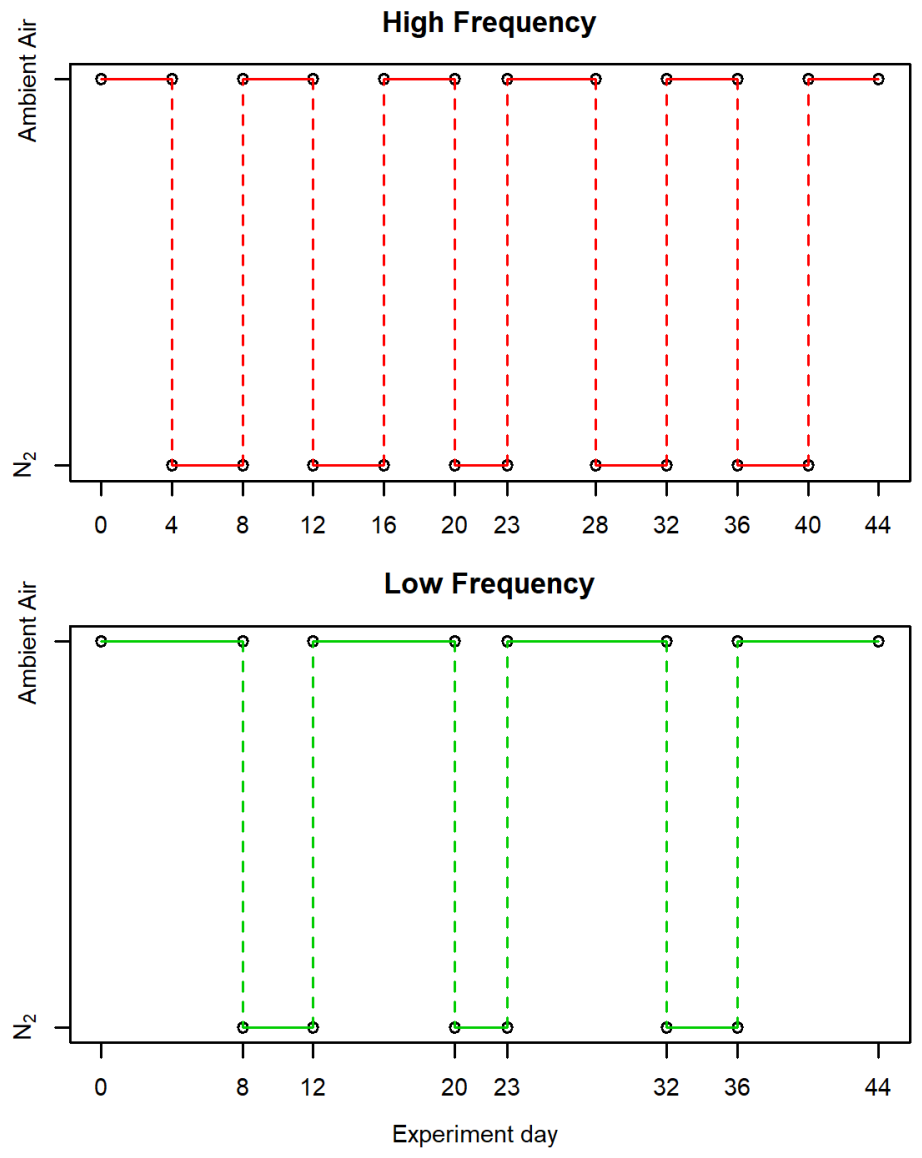

17

18 Fig. S1. Changes in headspace composition for samples from the two redox fluctuating 19 treatments. 


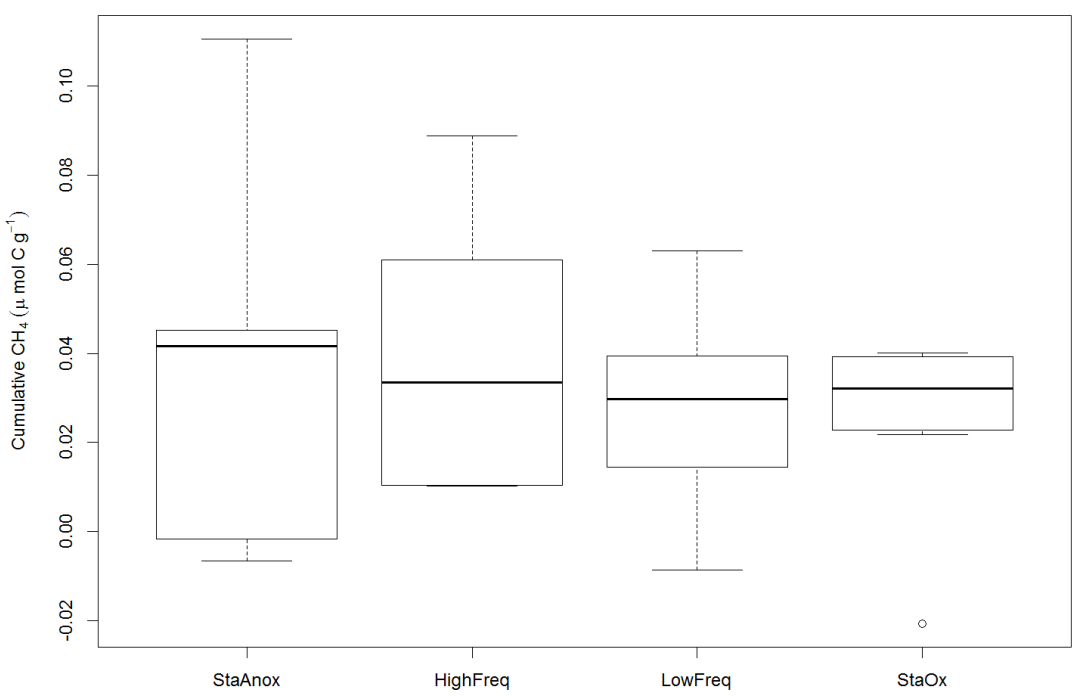

21

22 Fig. S2. Cumulative $\mathrm{CH}_{4}$ production over the incubation experiment.

23 

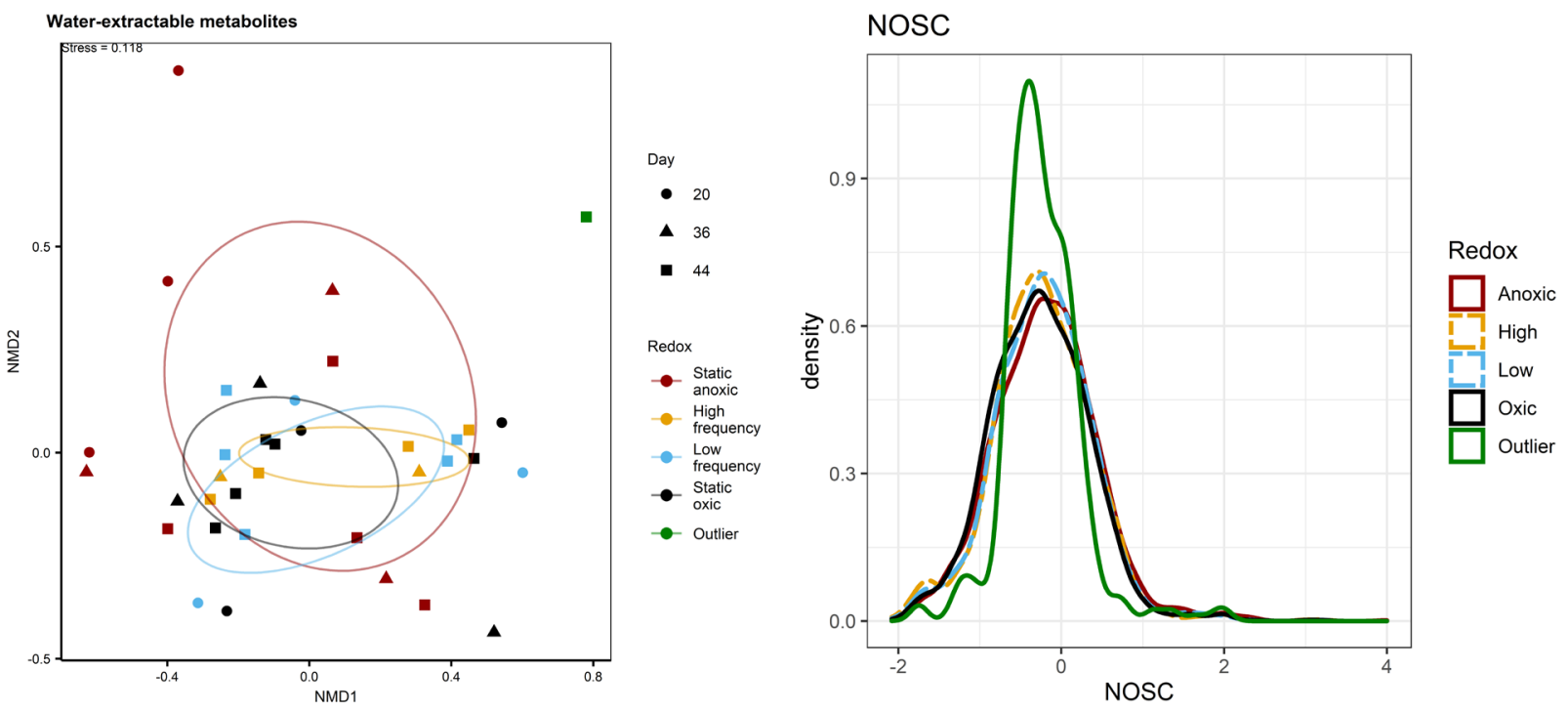

25 Fig. S3. (left) Non-metric multidimensional scaling (NMDS) plot comparing the composition of

26 water-extractable organic matter between the outlier and other samples. Data were derived from

27 FTICR-MS analysis. The eclipse indicates the standard deviation of each redox treatment. Note

28 the deviation of outlier from rest of the samples. (right) The probability density curves of NOSC

29 values of water-extractable organic matter comparing the outlier with other samples. Note the

30 large difference between the outlier and other samples and the small differences among redox

31 treatments. 
(a) Day 20

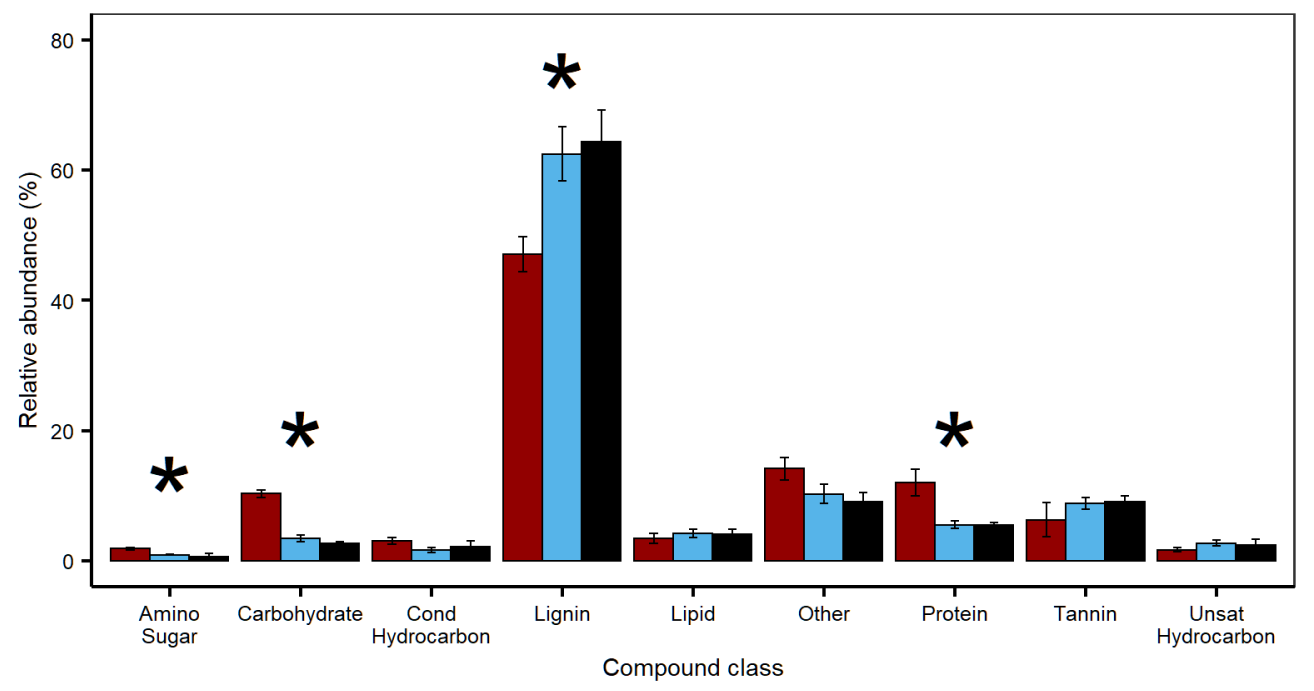

Redox treatment

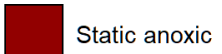
Low frequency Static oxic

(b) Day 44

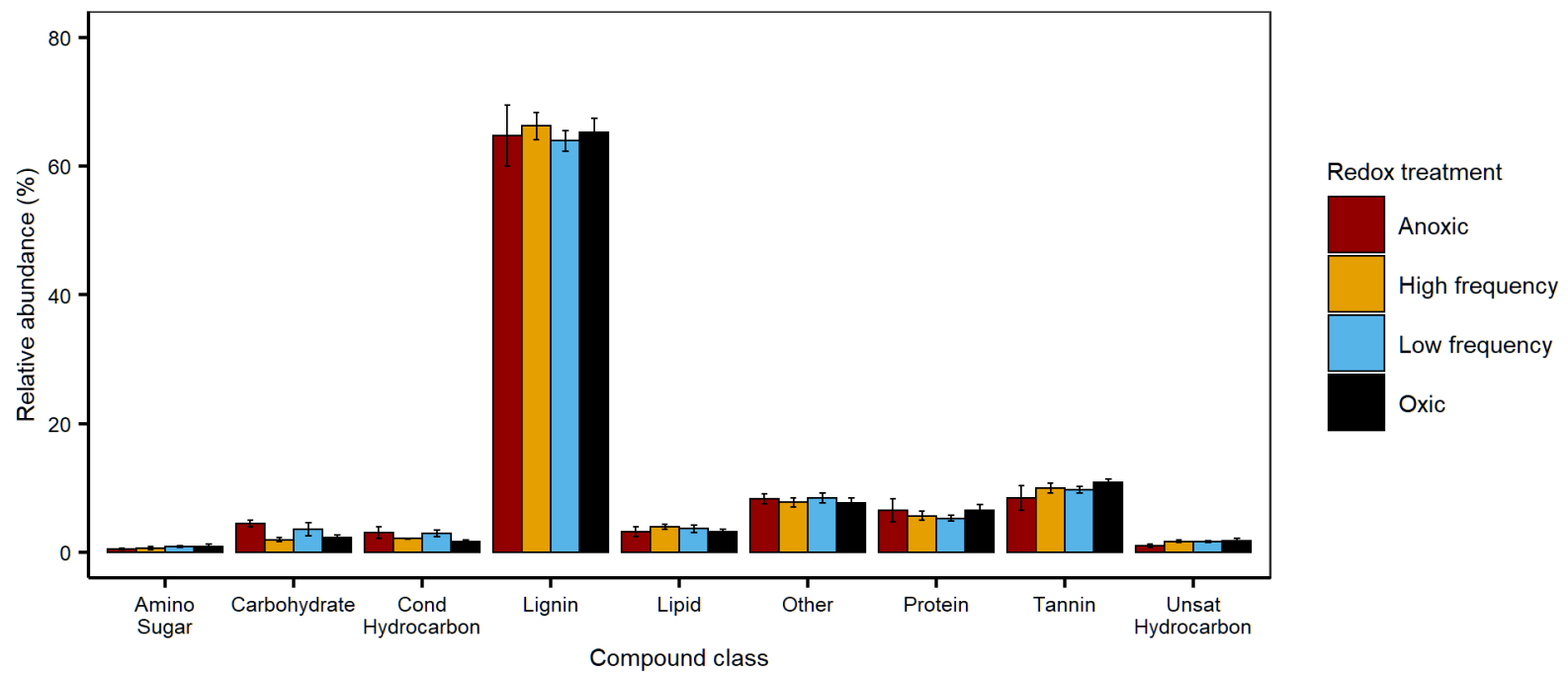

33 Fig. S4. Effects of four redox treatments on the relative abundances of chemical compound

34 classes extracted by water on day 20 (a) and day 44 (b) of a tropical soil incubation. Data

35 were derived from FTICR-MS analysis. Error bars indicate standard errors of means. *

36 indicate significant effects of redox treatments (ANOVA) at $\alpha=0.05$ level. On days 20 and

$3744, n=3$ and 5 per treatment, respectively. High frequency treatment from day 20 was not

38 included. Two outliers were removed from day 44. See the Materials and Methods for details. 


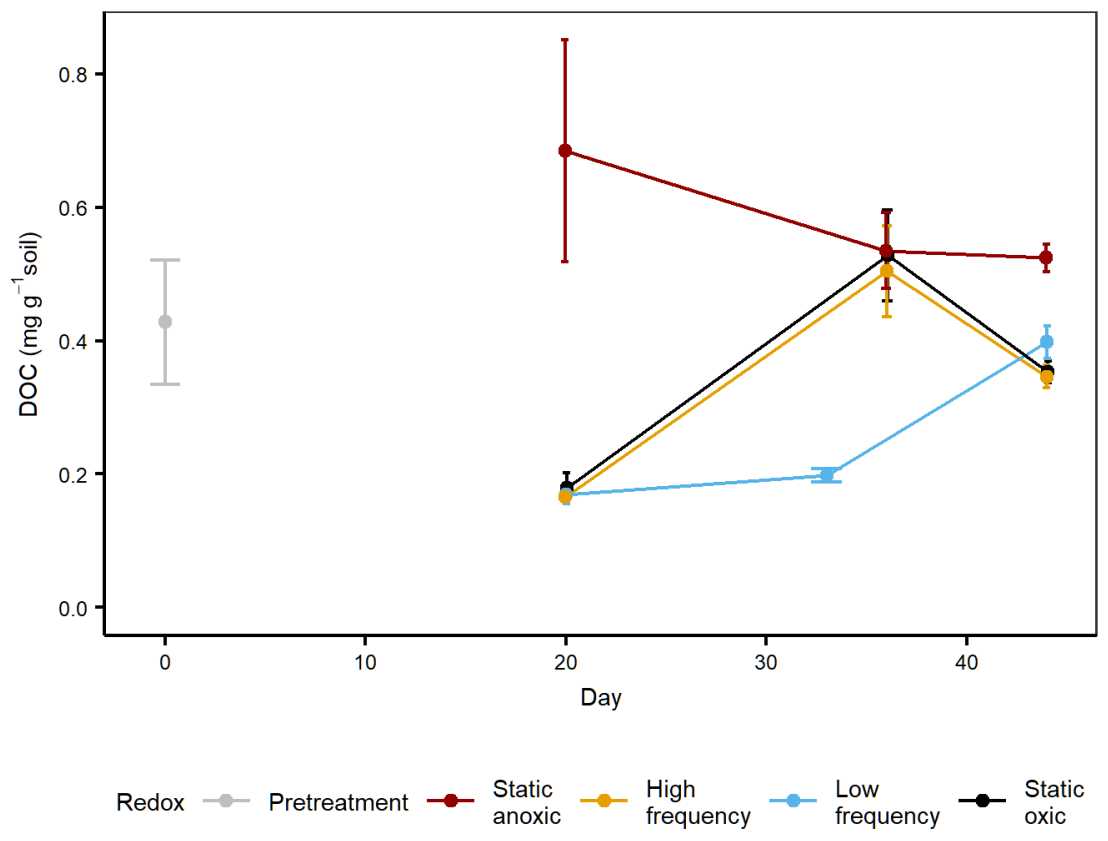

40

41 Fig. S5. Effects of redox treatments on the concentrations of water-extractable dissolved organic

$42 \mathrm{C}$ (DOC). Pretreatment values are in grey. $n=3$ per timepoint and treatment except $n=5$ on day

4344.

44 
45 Table S1. Carbon concentration and ${ }^{13} \mathrm{C}$ abundance of background soil and ryegrass litter (Mean

$46 \pm$ S.E.).

47

\section{C concentration $\quad{ }^{13} \mathrm{C}$ abundance}

\begin{tabular}{lll}
\hline Background soil $(\mathrm{n}=3)$ & $6.1 \pm 0.4 \%$ & $-29.0 \pm 0.8 \%$ \\
Ryegrass litter $(\mathrm{n}=6)$ & $41.0 \pm 0.1 \%$ & $96.7 \pm 0.1$ atom $\%$ \\
\hline
\end{tabular}

48

49 
50 Table S2. Total number of water-extractable molecular formulas identified by FTICR-MS

51 analysis per sampling days and treatments.

\begin{tabular}{lcccc}
\hline $\begin{array}{l}\text { Sampling } \\
\text { Day }\end{array}$ & $\begin{array}{c}\text { Static } \\
\text { anoxic }\end{array}$ & $\begin{array}{c}\text { High } \\
\text { frequency }\end{array}$ & $\begin{array}{c}\text { Low } \\
\text { frequency }\end{array}$ & $\begin{array}{c}\text { Stat52 } \\
\text { oxic } \\
53\end{array}$ \\
\hline 20 & 3795 & $584^{\mathrm{a}}$ & 2635 & $\begin{array}{c}2499 \\
54\end{array}$ \\
36 & 3070 & 1786 & $\mathrm{~N} / \mathrm{A}^{\mathrm{b}}$ & $\begin{array}{c}2888 \\
55\end{array}$ \\
44 & 3938 & 3394 & 4364 & 4697 \\
\hline
\end{tabular}

$57 \quad{ }^{a}$ Treatment was removed from further analysis due to the extremely low number of peaks 58 identified.

$59{ }^{\mathrm{b}}$ Treatment was not included in the original experimental design. 